\title{
Herbig Ae/Be Star Disks at High Angular Resolution
}

\author{
C.P. Dullemond \\ Max Planck Institut für Astrophysik, Postfach 1317, D-85741 Garching, \\ Germany \\ C. Dominik, R. van Boekel, R. Waters \\ Sterrenkundig Instituut 'Anton Pannekoek', Kruislaan 403, NL-1098 SJ \\ Amsterdam, The Netherlands \\ M. van den Ancker \\ European Southern Observatory, Karl-Schwarzschild Strasse 2, D-85748 \\ Garching bei München, Germany
}

\begin{abstract}
We show that there exists a simple geometric picture for the geometries of protoplanetary disks around Herbig Ae/Be stars that explains the two main kinds of spectral energy distributions found for these objects, and that makes predictions that are qualitatively in agreement with currently available spatially resolved images and/or interferometric measurements. Also it qualitatively explains the phenomenon of UX Orionis variability.
\end{abstract}

\section{Introduction}

Herbig Ae/Be stars are considered to be the intermediate mass counterparts of T Tauri stars (e.g. review by Waters \& Waelkens 1998). Due to their brightness, they are popular targets of high resolution studies of protoplanetary disks. Complete infrared spectra exist for many of them (van den Ancker et al. 2000; Malfait et al. 1999; Meeus et al. 2001), and resolved structure is observed at various wavelengths (see e.g. Monnier \& Millan-Gabet 2002; Leinert et al. this volume; Padgett et al. this volume).

In this contribution we want to show that a simple geometric picture exists that explains the spectral energy distributions (SEDs) of these objects, and is consistent with the resolved images at all currently probed wavelengths. In addition to this, the picture provides a natural explanation for UX Orionis variability.

The standard model for protoplanetary disks is that of a flaring disk which receives stellar radiation under a shallow grazing angle on its surface (Kenyon \& Hartmann 1987; D'Alessio et al. 1998; Chiang \& Goldreich 1997 henceforth CG97). While viscous dissipation may play a role in the innermost regions of the disk, the irradiation usually dominates the energy budget of the disk and thereby determines its structure. In principle this constitutes a complex 2-D axisymmetric 'radiation-hydrostatics' problem. But so far these equations were 
solved under the $1+1 \mathrm{D}$ splitting approximation. In this approximation the disk is assumed to be built out of mutually independent annuli, each representing a 1-D vertical structure problem. Such kind of models have been developed to various degrees of complexity by a number of authors (Calvet et al. 1991; D'Alessio et al. 1998; Bell et al. 1997; Malbet et al. 1991; Chiang \& Goldreich 1997; Dullemond et al. 2002; Lachaume et al. 2003).

While these models explain various features of infrared spectra of protoplanetary disks, such as the fact that dust features are usually in emission from such objects, they fail to explain the bump in the near infrared part of the SED observed for most Herbig Ae/Be stars. It was recognized by Natta et al. (2001) that dust evaporation may explain this feature. Dust evaporation removes the dust, and thereby the main opacity carrier, from the inner regions of the disk, leaving the optically thick dusty part of the disk with an inner rim at about 0.5 AU. This dust inner rim is directly exposed to the stellar radiation and thereby much hotter than the rest of the disk behind it. Since the temperature is set by the dust evaporation temperature $(1500 \mathrm{~K})$ this rim produces a thermal blackbody component in the SED in the near infrared. Dullemond, Dominik \& Natta (2001, henceforth DDN01) subsequently presented an improved version of the Chiang \& Goldreich model which includes the rim, and showed that the puffing up of the rim makes the near-infrared blackbody component strong enough to explain the near infrared bumps observed in most Herbig Ae/Be stars. In this paper it was also shown that the puffed-up inner rim casts a shadow over part of the disk behind it, while the flaring disk appears out of the shadow at roughly 5 to $10 \mathrm{AU}$ (Fig. 1A). Recently Muzerolle et al. (ApJ submitted) have studied the gas inward of the inner rim and confirmed that the gas is optically thin, or has such a small height that it casts only a very thin shadow over the inner dust rim. They also conclude that accretion luminosity can sometimes push out the radius of the inner rim (see D'Alessio this volume).

\section{A new geometry: self-shadowed disks}

The DDN01 model was succesfully applied by Dominik et al. (2003) to a sample of 13 Herbig Ae/Be stars for which the ISO SWS/LWS spectra were presented and studied by Meeus et al. (2001). It was shown that in most cases a reasonable fit to the SEDs could be made, but that sometimes the outer radius of the disk had to be taken very small, just outside the shadow of the inner rim. These turned out to be precisely the sources that were classified as 'group II' (not to be confused with Lada's class I/II classification) by Meeus et al. The classification by Meeus et al. divides the sources into those with strong far-infrared excess (group I) and those with weak far-infrared excess (group II). Since it is known from models of passive disks that the flaring outer parts of the disk are to be held responsible for any strong far-infrared excess, Meeus et al. proposed that the group I sources have flaring disks while the group II sources have flat disks. The fact that it were the group II sources that required such a very fine-tuned outer radius in the fits by Dominik et al. suggested that the flaring disk assumption of DDN01 may indeed be incorrect for these sources, in spite of the fact that they can be fitted by this model. 


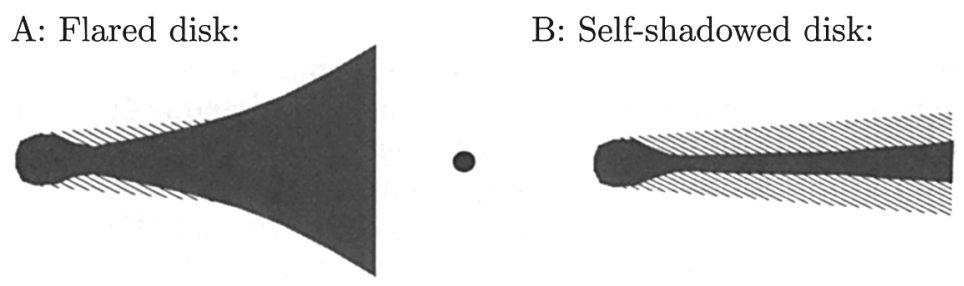

Figure 1. Pictographical illustration of the two geometries that follow from the 2-D axisymmetric modeling.

Independently of this, in an attempt to better treat the shadowed regions in the DDN01 model, an effort was undertaken to model the disks with 2-D axisymmetric radiative transfer (i.e. 3-D transfer but without any dependence of the variables on the $\Phi$ coordinate). New radiative transfer techniques were developed to overcome the difficulties of the extreme optical depths in these disks (up to $\tau=10^{7}$ in some cases). By coupling the radiative transfer calculations to the equations of vertical hydrostatic equilibrium we obtained self-consistent 2-D axisymmetric disk models for Herbig Ae/Be stars (Dullemond 2002; Dullemond \& Dominik A\&A submitted). It was found in these papers that in addition to the "flaring disk with rim and shadow" geometry of DDN01, there exist solutions in which the shadow extended over the entire disk (see Fig. 1B). Typically these solutions appear when the disk is less massive, or when dust grains have grown and thereby have a reduced opacity. Also dust settling could cause flared disks to transform into self-shadowed disks. These "self-shadowed" disks have no flaring outer part and therefore are not heated by direct irradiation. Indirect irradiation by the hot upper and lower parts of the inner rim and by radial radiative diffusion prevent the disk from cooling down to the temperature of the molecular cloud. The shadowed outer parts are much cooler than the flaring disks, but due to this indirect irradiation they are still capable of producing non-negligible far-infrared emission, albeit much weaker than the far-infrared emission from flared disks.

From these models it followed that the flaring disk solutions produced SEDs very similar to the group I sources, while self-shadowed disk solutions produced SEDs similar to the group II sources. It is therefore natural to associate these geometries to these groups. And it is tempting to set this in an evolutionary scenario where one starts out with massive flaring disks with small grains (group I), and as the grain coagulate and settle, or the disk evaporates, the disk becomes self-shadowed (group II). But before reaching such conclusions it is important to test the "group I = flaring / group II = shadowed" hypothesis. One way to do this is by high angular resolution observations. 


\section{Testing the models: high-resolution imaging}

One prediction by the models is that group II sources should not be seen (or at least be faint) in scattered light images in the optical and near-infrared, because self-shadowed disks do not have a scattering surface exposed to the stellar light (see Fig. 1B) as opposed to the group I flared disks. Tentative results seem to confirm this. While group I sources like AB Aurigae and HD100546 have been imaged at optical wavelength as strong scattering nebulae (Grady et al. 1999; Augereau et al. 2001), there have been unexplained "non-detections" in scattered light for HD104237, MWC 480, HD 142666 (private communication C. Grady, Augereau et al. 2001), even though in many of these cases the disk has been detected and resolved in CO lines (Mannings \& Sargent 1997, 2000). As it turns out, these sources are indeed classified as group II, confirming the predictions. An intermediate case HD163296 (on the border between I and II) shows some nebulosity, though rather weak (Grady et al. 2000).

Another way to test the flaring/self-shadowed hypothesis is by its predictions for mid-infrared interferometry. One of the predictions is that the visibility function is constant between $9 \mu \mathrm{m}$ and $13 \mu \mathrm{m}$, and that it rises when one goes to wavelengths shorter than about $9 \mu \mathrm{m}$. The reason is that longward of $9 \mu \mathrm{m}$ the SED is dominated by the emission from the surface layer of the disk (which is a comparatively large source), while shortward of $9 \mu \mathrm{m}$ the emission from the inner rim (which is a compact source) becomes important. Leinert et al. (this volume) show first results of the VLTI MIDI instrument obtained just before this conference. They indeed show the predicted rise shortward of $9 \mu \mathrm{m}$. The precise value of the visibility functions is not a perfect match (about $50 \%$ off), but considering the blind fitting strategy (the models were from the SED fitting by Dominik et al.) we believe that this is still reasonably successful.

Near-infrared interferometry enables us to test the hypothesis of the puffedup inner rim. Using aperture masking interferometry with the Keck, Tuthill et al. (2001) discovered a half-moon like emission around the very bright Herbig Be star $\mathrm{LkH} \alpha$ 101, which they interpreted (independently from Natta et al. 2001) as the inner rim of a disk in which dust evaporation removed the dust from inner part of the disk. The radius measured for this ring seems to be consistent with the dust evaporation hypothesis. Millan-Gabet et al. (2001) and Eisner et al. (2003) show the existence of similar structures around many Herbig Ae stars. On average, the radii of these rings seems to agree reasonably well with the dust sublimation radius (Monnier \& Millan-Gabet 2002).

\section{UX Orionis Variability}

The flaring/self-shadowed picture also seems to be able to qualitatively explain the behavior of UX Orionis type stars in which patches of dust passing in and out of our line of sight toward the star cause the star to vary in brightness on a time scale of days to weeks. It has been suggested by a number of authors (e.g. Grinin 1991; Bertout et al. 2000) that a nearly-edge-on disk could explain the extinction events: hydrodynamic perturbations every now and then throw up blobs of gas and dust into the line of sight as it skims the surface of the disk. The problem with this scenario is that for flaring disks only the outer 
regions of the disk can be responsible for these events. This would result in events lasting years rather than days. The inner rim of the model we propose seems a much more likely candidate, as the inner rim is located at about 0.5 AU. But for this to work the line of sight to the star must skim the upper part of the puffed-up inner rim. Only for self-shadowed disks this is possible without huge extinction from the outer parts of the disk. This makes the prediction that UXORs should belong to group II. Dullemond et al. (2003) provide strong observational evidence confirming this model prediction.

\section{Conclusion}

We conclude that there is now significant evidence for the identification of flaring/self-shadowed disk geometries with Meeus et al. group I and group II sources, respecively. We speculate that an evolutionary link from group I to group II may exist through the onset of dust coagulation/settling and/or disk dispersal.

\section{References}

Augereau, J. C., Lagrange, A. M., Mouillet, D., \& Ménard, F. 2001, A\& A, 365, 78

Bell, K. R., Cassen, P. M., Klahr, H. H., \& Henning, T. 1997, ApJ, 486, 372

Bertout, C. 2000, A\& A, 363, 984

Calvet, N., Patino, A., Magris, G. C., \& D'Alessio, P. 1991, ApJ, 380, 617

Chiang, E. I. \& Goldreich, P. 1997, ApJ, 490, 368

D'Alessio, P., Canto, J., Calvet, N., \& Lizano, S. 1998, ApJ, 500, 411

Dominik, C., Dullemond, C. P., Waters, L. B. F. M., \& Walch, S. 2003, A\& A, 398,607

Dullemond, C., van den Ancker, M., Acke, B., \& van Boekel, R. 2003, ApJL, in press

Dullemond, C. P., Dominik, C., \& Natta, A. 2001, ApJ, 560, 957

Dullemond, C. P., van Zadelhoff, G. J., \& Natta, A. 2002, A\& A, 389, 464

Dutrey, A., Guilloteau, S., Prato, L., Simon, M., Duvert, G., Schuster, K., \& Menard, F. 1998, A\& A, 338, L63

Eisner, J. A., Lane, B. F., Akeson, R. L., Hillenbrand, L. A., \& Sargent, A. I. 2003, ApJ, 588, 360

Grady, C. A., Devine, D., Woodgate,B. et al., 2000, ApJ, 544, 895

Grady, C. A., Woodgate, B., Bruhweiler, F. C., Boggess, A., Plait, P., Lindler, D. J., Clampin, M., \& Kalas, P. 1999, ApJL, 523, L151

Grinin, V. P., Kiselev, N. N., Chernova, G. P., Minikulov, N. K., \& Voshchinnikov, N. V. 1991, Astrophysics \& Space Science, 186, 283

Kenyon, S. J. \& Hartmann, L. 1987, ApJ, 323, 714

Lachaume, R., Malbet, F., \& Monin, J.-L. 2003, to be published in A\& A

Malbet, F. \& Bertout, C. 1991, ApJ, 383, 814 
Malfait, K., Waelkens, C., Bouwman, J., De Koter, A., \& Waters, L. B. F. M. 1999, A\& A, 345, 181

Mannings, V. \& Sargent, A. I. 1997, ApJ, 490, 792

Mannings, V. \& Sargent, A. I. 2000, ApJ, 529, 391

Meeus, G., Waters, L. B. F. M., Bouwman, J., van den Ancker, M. E., Waelkens, C., \& Malfait, K. 2001, A\& A, 365, 476

Monnier, J. D., Millan-Gabet, R., 2002, ApJ, 579, 694

Millan-Gabet, R., Schloerb, F. P., \& Traub, W. A. 2001, ApJ, 546, 358

Natta, A., Prusti, T., Neri, R., Wooden, D., \& Grinin, V. P. 2001, A\& A, 371, 186

Tuthill, P. G., Monnier, J. D., \& Danchi, W. C. 2001, Nature, 409, 1012

van den Ancker, M. E., Bouwman, J., Wesselius, P. R., Waters, L. B. F. M., Dougherty, S. M., \& van Dishoeck, E. F. 2000, A\& A, 357, 325

Waters, L. B. F. M. \& Waelkens, C. 1998, ARA\&A, 36, 233 